\title{
Malaria Treatment at Community; Can Rapid Diagnostic Test Eliminate Stock-out of Artemisinin-Based Combination Therapy in South Sudan
}

\author{
Article by Dricile Ratib \\ PhD, Public health, Texila American University, Uganda \\ E-mail:dricileratib@yahoo.co.uk
}

\begin{abstract}
Malaria remains endemic in South Sudan, accounting for $20 \%$ of deaths and $30 \%$ of hospital admissions (Global Fund, 2015). The 2014 to 2021 South Sudan national malaria strategic plan aims to bring down the morbidity and mortality related to malaria by $80 \%$, but stock-out of drugs is a major challenge (National malaria control program [NMCP], 2017) ${ }^{l}$.

South Sudan received over fifty five million (55,585,832 USD) United states dollars from Global fund in 2015 for fighting malaria in addition to similar amounts from Department of International development (DFID) but this has not changed the ant-malaria stock out status(DFID, 2015). In the last quarter of 2014, 854 Community based distributors (CBDs) from two sub-recipients had stock out of Artemisinin based combination therapy (ACTs) (Global Fund, 2015) ${ }^{2}$.

This retrospective study re-analyzed the data used for training 240 community based drug distributors (CBDs) in April 2015 on the use of malaria rapid diagnostic test (RDT). Of those who passed the post test, 131 were given kits of RDT to practice the skills they had acquired and give ACTs only to the children 2-59 months who test positive for malaria.

The number of children assessed with fever were 1047, (534 males and 513 females); 1012 were done RDT and only 364(36\%) tested positive for plasmodium falciparum malaria. The iCCM programme in South Sudan treats all fever cases with ACTs. This one month RDT use therefore saved $64 \%$ of the ACTs that would have been irrationally used.
\end{abstract}

\section{Introduction}

\section{Problem statement}

The acute on chronic conflict in South Sudan has destroyed and retarded the health systems to fight malaria, a serious acute infectious illness spread by bites of infected female anopheles mosquito, and is the leading cause of morbidity and mortality in the country, which accounts for $20-40 \%$ of morbidity resulting in over $20 \%$ of deaths reported at health facilities and $30 \%$ of all hospital admissions $^{3}$. The 2009 malaria indicator survey showed that prevalence of infection in children Under 5 was $24.5 \%$ (95\% CI: 23.0-26.1) and in pregnant women was 9.9\% (95\% CI: 7.4-13.1). About two thirds (64\%) of children under five (5) and $46 \%$ of pregnant women were anemic (Eyobo et al $2014)^{4}$.

The ministry of health has developed national malaria control strategy 2015-2021 that lays down a framework for the prevention, diagnosis, treatment and control of malaria in the Country (WHO Afro $2017)^{5}$. The key interventions are information, education and behaviour change communication programmes; early detection with rapid diagnostic tests (RDTs), treatment with artemisinin-based combination therapy (ACT), indoor residual spraying (IRS), use of long-lasting insecticidal nets (LLINs) and intermittent preventive treatment (IPT) for pregnant women. The South Sudan MoH Malaria strategy is in line with the WHO Global Technical Strategy for Malaria (2016-2030) ${ }^{6}$,

\footnotetext{
${ }^{1}$ NMCP South Sudan -National malaria strategic plan 2014-2021

2 OIG_GF-OIG-15-016_Report_en.pdf

${ }^{3}$ World malaria report 2017

${ }^{4}$ Malaria indicator survey 2009, South Sudan: baseline results at household level

${ }^{5}$ south-sudan-national-malaria-strategic-plan-2014-2021-launched

${ }^{6}$ Global technical strategy for malaria 2016-2030
} 
health sector development plan 2012-16 (MoH- South Sudan 2012) ${ }^{7}$ and Action and Investment to defeat Malaria (2016-2030) strategy developed by the Roll Back Malaria (RBM) partnership (WHO, 2016) ${ }^{8}$.

The World health organization (WHO) recommends parasitological confirmation of malaria through quality assured diagnosis, in all settings before treatment is started (WHO 2010) ${ }^{9}$. Treatment solely on the basis of clinical suspicion should only be considered when a parasitological diagnosis is not accessible (WHO 2011) ${ }^{10}$. Prompt diagnostic confirmation of malaria can be achieved through good quality microscopy. Since this is not feasible in many rural settings across Africa, especially South Sudan, quality assured malaria Rapid Diagnostic Tests (RDTs) represent suitable alternatives for confirmation of malaria diagnosis prior to treatment at the health facilities (MoH- South Sudan National Malaria control program 2015) ${ }^{11}$.

However, the situation is not the same at the community level where integrated community case management of malaria takes place. Government of South Sudan through support from partners has been implementing a home management of malaria as part of the integrated community case management of malaria, pneumonia diarrhoea and malnutrition (iCCM) program in the country including the counties like Aweil centre and Aweil west of Northern Bahr el Ghazal state since 2010 which is shown to reduce child mortality by $40 \%$ (Population services international [PSI] 2012, Malaria Consortium $[\mathrm{MC}], 2012)^{12} \cdot{ }^{13}$ The program targets children between 2-59 months and treatment are conducted by community based distributors (CBDs). Treatment of malaria at community level by community based distributors (CBDs) in these areas has been solely based on clinical suspicion due to the inaccessibility of parasitological diagnosis leading to inclusion of significant proportion of febrile illnesses that are not necessarily malaria who needed to be assessed for other causes of fever and given appropriate treatment(WHO 2012) ${ }^{14}$. The result is chronic stock out of ACTs especially during the peak of rainy season when ACTs are needed most; hence the impact of the project is not being felt as it should due to the stock-outs constraints (Systems for improved access to pharmaceuticals and services [SIAPS]2016, 2015),${ }^{15}{ }^{16}$.There was need therefore to find out what proportion of the ACTs are being given to children with febrile illness who do not actually have malaria by reviewing and re-analyzing available RDT and ACT data from a pilot study done in Northern Bahr el Ghazal in 2015.

\section{Main objective}

The purpose of the study is to find out if the irrational use of ACTs for all children 2-59 months with febrile illness contributes to the chronic stock out of ACTs in former Northern Bahr al Ghazal state of South Sudan.

\section{Specific objectives}

- To find out the proportion of children 2-59 months with fever who tested positive for malaria with rapid diagnostics tests (RDTs) in Aweil Centre and Aweil West Counties in April 2015

- To find out the doses of ACTs given to children 2-59 months during the period when RDT was used in Aweil Centre and Aweil West Counties in April 2015

\footnotetext{
${ }^{7}$ Health-sector-development-plan-2012-2016.pd

${ }^{8}$ Malaria Policy Advisory Committee to the WHO: conclusions and recommendations of eighth biannual meeting (September 2015)

9 9789241599412_eng.pdf-parastological confirmation of malaria diagnosis

${ }^{10}$ world_malaria_report_2011/9789241564403_eng.pdf

${ }^{11}$ Approved-Printed-Version_SSD-MCM-Guidelines_2015.pdf

12 iCCM Program Brief Oct 2012_5__1

${ }^{13}$ Facilitator-Training-Manual-iCCM-South-Sudan_Malaria-Consortium_2012

${ }^{14}$ Training module on malaria control : case management

${ }^{15}$ Malaria-pmi-quarterly-updates-april-june-2015

16 pdf.usaid.gov/pdf_docs/PA00MW5Q.pdf
} 


\section{Methodology}

\section{Study area}

The malaria RDT field test was conducted in Aweil centre and Aweil west counties of former Northern Bahr el Ghazal state in April 2015. Northern Bahr el Ghazal was one of the 10 states of South Sudan. It had an area of $30,543 \mathrm{~km}^{2}$ and is part of the Bahr el Ghazal region (National Bureau of statistics [NBS], 2011)17. The state is susceptible to flooding which creates a contusive environment for mosquito breeding resulting in the state having high malaria incidences during the rainy season(Magda Armah Penn Amaah and Jemila M. Ebrahim, 2016 )18. Flooding commonly occurs in the later part of the rainy season, from July to December, and is known to cause issues with accessibility. The two counties covered by the operational research: Aweil centre and Aweil west are also flood-prone areas with silty soils and poor drainage system19 (Max Gibson and Eric Kramak 2016).

\section{Sampling method}

Two Payams were randomly selected one from each of the Counties, Aweil centre and Aweil west. All the 240 CBDs and their supervisors in the selected payams of Gomjuer east and Chel south were included in the malaria RDT field test. Selected CBDs had different education levels. A total of $46.7 \%$ were illiterate and $40 \%$ and $13.3 \%$ had reached elementary and high school level respectively. The majority $85 \%$ had at least one year ICCM experience.

The 240 CBDs and CBD supervisors participated in one day training on malaria RDT use. The main objective of the training was to test whether a minimal investment in training time and use of simple job aid could achieve satisfactory competency. Supplies used during the training included RDTs, gloves, alcohol swabs, lancets, sharps and non-sharps bins, and a copy of the job aid for each participant.

The training was facilitated by Ministry of health officials from the County health department (CHD) and technical team from health partner. The training had two sections the theoretical and practical part. The generic WHO malaria RDT job aid was simplified and used during the training (WHO 2010) $)^{20}$.

The facilitators started by reviewing patient history taking and physical examination. This was to ensure that only children with fever were tested for malaria with RDT. They then demonstrated step by step how to conduct malaria RDT starting with how to open the test packet, assemble the RDT items, conduct the test, interpret and record the results. The training materials were revised to be more pictorial with less writing to enable the CBDs who are illiterate to benefit too. The trainees then practiced on one another and were given assistance by the trainers who attended the trainings. A pre and post-test was administered together with a competency checklist to ensure that the competency of the trainees was confirmed before they could go and administer RDT on actual patients. Only the CBDs who passed the competency test were allowed to proceed to test children with fever and those found positive for malaria were given ACTs according to the iCCM guidelines (WHO and UNICEF $2012)^{21}$.

\footnotetext{
${ }^{17}$ key_indicators_for_northern_bahr_el_ghazal.pd

18 health-cluster-bulletin_24-august---06-sept-2016.pd

${ }^{19}$ Understanding flood resilience in rural South Sudan

${ }^{20}$ Generic_PfPan_training_manual_web.pdf

${ }^{21}$ WHO/UNICEF JOINT STATEMENT - Integrated Community Case Management: An equity-focused strategy to improve access to essential treatment services for children
} 


\section{Sample size and sampling design}

This was a retrospective cohort study. Two Payams were randomly selected one from Aweil Centre and another from Aweil West. All the children who presented with fever to the CBD from the selected payams were enrolled into the study. A total of 1047 children 2-59 months who had fever from the two payams of Chel South and Gumjur east of Aweil centre and Aweil west respectively were enrolled into the study. Of these 513 were females and 534 were males. Of these 996 were tested for malaria with RDT. And those found to be positive were give Artemisinin-based Combination Therapy for treatment of malaria by the community drug distributors.

\section{Research team}

The research team included the 131 community based drug distributors (CBDs) out of 240 CBDs who were trained and passed the post test well, (42 from Gumjur east and 89 from Chel South Payams) of Aweil west and Aweil centre respectively); 16 CBD supervisors half from each of the two Payams, two field officers one for each Payam, Monitoring and Evaluation officer and the researcher.

The function of the CBDs was to screen children 2-59 months for fever who were then assessed for danger signs like anemia, convulsions, severe acute malnutrition. Those with fever were done RDT while those with danger signs were referred and those with fever who were well outside the age bracket were reassured and sent back home. The CBD also recorded the results of the RDT tests. Those who tested positive were given ACT and those with fever, but tested negative were given amoxicillin according to the iCCM guidelines with suspicion that they could be having bacterial infection commonest in the area like streptococcus are responsive to amoxicillin. The function of the CBD supervisor was to oversee 10-15 CBDs weekly on bicycle to see if they were working and the records were well made in the patient register. He made corrections where necessary and summarized the results from his CBDs into caseload summary. The field officer rode on motor bike to supervise the CBD supervisors and collects the caseload summaries to be handed over to the $\mathrm{M}$ and $\mathrm{E}$ officer who then made entries into the data base made by the researcher.

\section{Research equipment and materials}

For the training of the CBDs and all the research team, standard WHO training materials on malaria rapid diagnostic tests were used (WHO 2010) ${ }^{22}$. 8Bicycles for CBD supervisors, 2motorbikes for field officer, and a vehicle hire for the researcher, desk top computer for $\mathrm{M}$ and $\mathrm{E}$ officer, a laptop for the researcher. The ministry of health, republic of South Sudan patient register was used to capture the patient information and CBD supervisor case load summary for the summarizing the data from 1015 CBDs.

\section{Data collection}

Since the data for the CBDs is being recorded in the ICCM registers, recorded in the iCCM registers, especially CBD patient registers designed by the ministry of health, the data collectors needed only the period of the data collection which was April 2015 for which the RDT pilot and training was conducted. The original purpose of the RDT pilot was to assess whether the CBDs some of whom were illiterate could be able to gain competency and skills during the training to be able to do the RDT tests. In this retrospective study, the focus is the RDT results and the ACTs used, hence different objectives and data actually required.

The hard copies of CBD patient registers are kept in the office of the implementing partner in Aweil state capital. The filling is stratified by Payam, hence this made it easier for the data collectors to locate the files for the CBDs from Chel south and Gumjur east payams for the period of April 2015. The data is then entered in to the data-base developed by the researcher and then analyzed.

\section{Quality assurance}

To ensure that the tests and treatments given by the CBDs are reliable, all the CBDs were trained $1^{\text {st }}$ for 6 days based on the ministry of health ICCM guidelines then for one additional day with focus

${ }^{22}$ Generic_PfPan_training_manual_web.pdf 
on RDT use making a total of one week training. Only those CBDs who pass the competence test were given RDT to test the children. A maximum of 15 CBDs were supervised by a CBD supervisor to ensure that the quality of supervision is good and enough time is taken by the CBD supervisor to check the RDT cassettes already used and compared with the results recorded by the CBD in the patient register. The ACT treatment is also checked for the storage conditions, expiry dates, and stock records and compared with ACTs issued out. The researcher did spot checks from sample CBD registers and $\mathrm{CBD}$ supervisor case load summery and compared this with the $\mathrm{M}$ and $\mathrm{E}$ data in soft copy for consistency.

\section{Data analysis}

Data analysis was done using statistical package for social scientists (SPSS). This is then compared for consistency with the data in the district health information system (DHIS) which is an access based software used by the ministry of health in South Sudan for data entry and analysis.

\section{Results}

The total number of children with fever assessed by the 131 community based drug distributors (CBDs) for a period of 1 month (April 2015) was 1047. Of these 534 were boys and 513 girls. 841 of the children were from Aweil Centre while 206 were from Aweil west. Not all the children were tested, only 1012 out of 1047 were tested with rapid diagnostic test (RDT); of these 364 (36\%) tested positive for falciparum malaria. All the children who tested positive for RDT were given ACT, 71 were infants given ACT infant while 349 were toddlers given ACT toddler. 25 children (20 from Aweil Centre and 5 from Aweil west who had fever were not tested for malaria, but were given ACTs (see table1) 


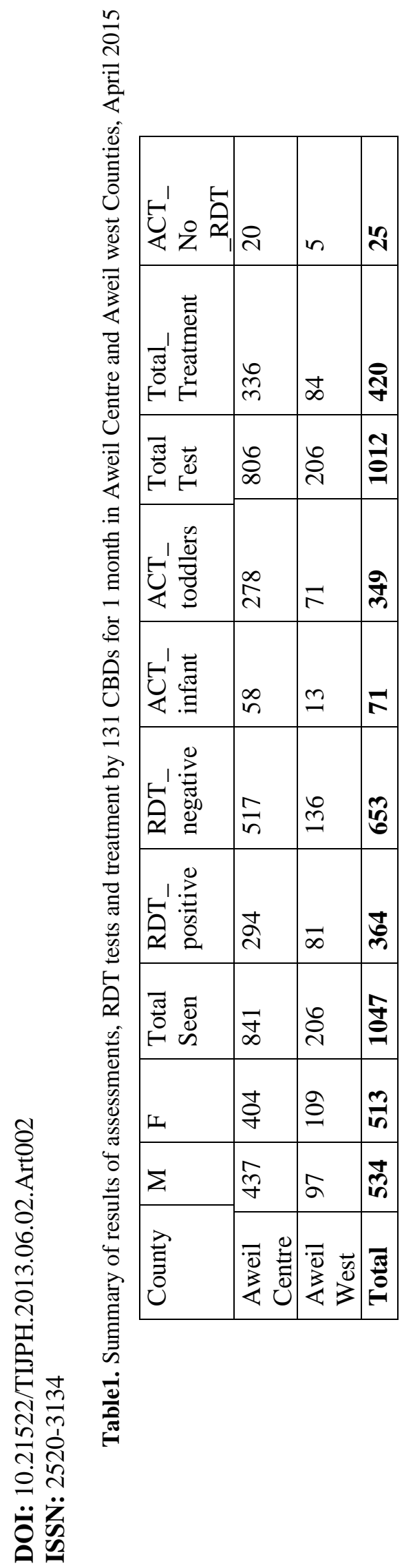


During the period $1^{\text {st }}$ to $30^{\text {th }}$ April 2015, 131 CBDs assessed 1047 children with fever. This gives a mean of 8children with fever cases per CBD. Since only $36 \%$ of these children assessed with fever tests positive for malaria with RDT, the mean number of ACT doses issued out per CBD is 3 doses per month (see table 2).

Table2. Mean malaria caseload for CBDs for the 1month period April 2015

\begin{tabular}{|l|l|l|l|l|l|}
\hline \multicolumn{7}{|l|}{ Descriptive statistics-CBD caseload } \\
\hline & N & Minimum & Maximum & Mean & Std. Deviation \\
\hline $\begin{array}{l}\text { No. of children tested per } \\
\text { CBD }\end{array}$ & 131 & 1.00 & 12.00 & 7.9924 & 3.03187 \\
\hline $\begin{array}{l}\text { ACT doses given per } \\
\text { CBD }\end{array}$ & 131 & .00 & 10.00 & 3.2061 & 2.73079 \\
\hline Valid N (list wise) & 131 & & & & \\
\hline
\end{tabular}

The number of boys and girls who suffered from malaria during the research period was 534 and 513 respectively. These constitute $51 \%$ and $49 \%$ with boys tending to have more illness than girls (See fig: 2 below).

\section{Percentage of children with fever by gender in Aweil Centre and Aweil West April 2015}

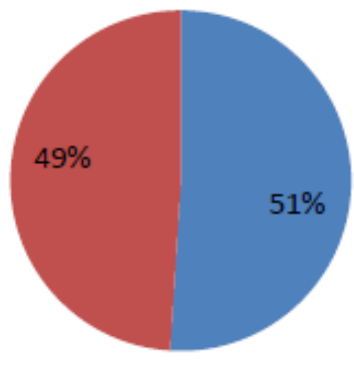

Figure 1. \% Distribution of children assessed with fever by gender-Aweil Centre and Aweil West Counties April 2015.

Over one thousand (1047) children were assessed with fever and 1012 tested with RDT for falciparum malaria, the most common species of plasmodium in the republic of South Sudan. Of those tested only $420(36 \%)$ tested positive for malaria and were given AC treatment (table 3). However, in the integrated community case management of malaria (iCCM), all children with fever are treated with ACT (MoH 2010). This means that if the for the month of April alone 2016, 627 doses of ACT would be lost to irrational use in the community through the community based treatment programme. Of the 627 ACTs saved, 505 (60\%) were from Aweil Centre and 122 (59\%) from Aweil West. The mean ACTs saved for the two counties in April 201 was $59.6 \%$ ( see fig 3 below).

Table 3. No. of ACTs saved through RDT use in aweil centre and aweil west april, 2015.

\begin{tabular}{|l|l|l|l|l|}
\hline County & Children with fever & ACT Treatments & ACTs saved & \% ACTs saved \\
\hline Aweil Centre & 841 & 336 & 505.0 & $\mathbf{6 0 . 0}$ \\
\hline Aweil West & 206 & 84 & 122.0 & $\mathbf{5 9 . 2}$ \\
\hline Total & $\mathbf{1 0 4 7}$ & $\mathbf{4 2 0}$ & $\mathbf{6 2 7 . 0}$ & $\mathbf{5 9 . 9}$ \\
\hline & & & & \\
\hline
\end{tabular}




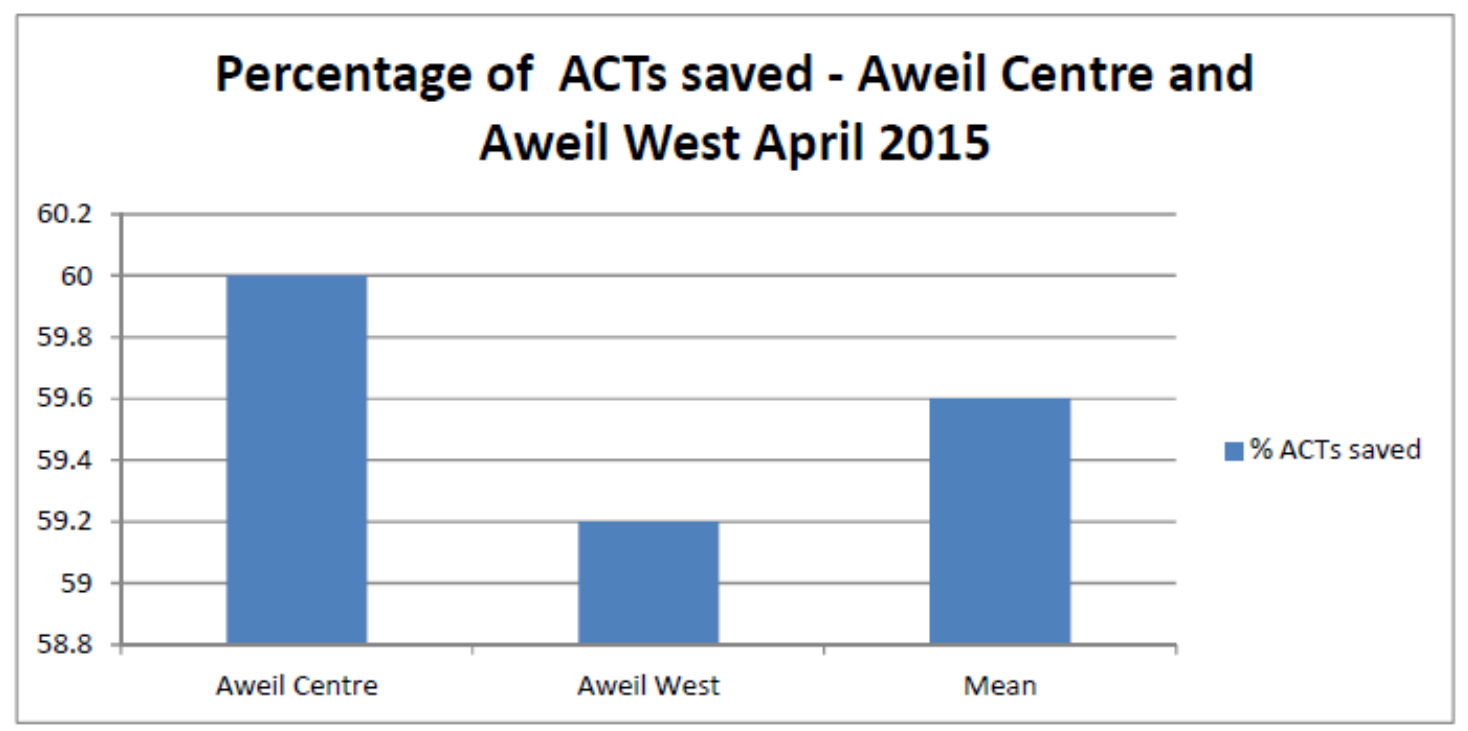

Figure 2. Mean ACTs saved from Aweil Centre and Aweil west Counties April 2015

\section{Discussion}

The ministry of health, UN agencies like WHO and UNICEF international non-governmental organizations and community based organizations among others have being trying hard to reduce morbidity and mortality related to malaria, especially among children, but this has not made significant impact as infant and young child mortality related to Malaria remains high especially during the rainy season partly due to ACT stock-outs. In 2017, 1.3 million people were infected in South Sudan and 220 died every week (ALIMA 2017) ${ }^{23}$

The findings of this retrospective study show that, only $36 \%$ of the fever cases being treated with ACT are actually caused by plasmodium falciparum. This result answers the research question in the objectives and is consistent with the study done in 2013 for the national malaria indicator survey which showed that $30 \%$ of all hospital admissions were due to Malaria (Eyobo B. M, 2014) ${ }^{24}$. This result is consistent with other similar study results from Tanzania which showed only $50 \%$ of fevers among children tested positive for malaria (Mubi et al 2011) 25 $^{25}$ and 58.6\% in Myamar (Ohnmar et al 2012). ${ }^{26}$ This has been partly attributed to frequent drug stock outs at all levels in the health care structure up to the community based programmes like iCCM(Dick, M., Farai, C., \&Joseph, N. $2011)^{27}$. Up to $27 \%$ of the drug stores, health facilities and pharmacies experience stock out of medicines in South Sudan (USAID, 2011). ${ }^{28}$

This study was done in April which is not the peak of malaria season; it is likely that the figures would be higher if the study was done in the months of August to November when the rains and subsequent flooding provides heavens for the breeding of mosquitos in Northern Bahr el Ghazal. The question now is what is the cause of 64\% of fever cases among children 6-59 months of age for which ACTs have been irrationally used?

The integrated community case management of malaria pneumonia and diarrhea in South Sudan and home based management malaria in the neighboring Uganda share one thing in common; they all suffer from chronic stock out of ACTs despite millions of dollars spent on procurement of ACTs

\footnotetext{
${ }^{23}$ ALIMA 2017, South-Sudan-mobile-clinics-respond-malaria-peak, para 4

${ }^{24}$ Malaria indicator survey 2009, South Sudan: baseline results at household level

${ }^{25}$ Malaria Rapid Testing by Community Health Workers Is Effective and Safe for Targeting Malaria Treatment: Randomised Cross-Over Trial in Tanzania.
}

${ }^{27}$ Pharmaceutical logistics assessment in South Sudan, Global health and technical assistance project

${ }^{28}$ Pharmaceutical logistics assessment in South Sudan, Global health and technical assistance project 
which are ideally enough to treat the $36 \%$ of the population with Malaria. Due to the frequent stock out of ACTs, some families in Uganda prefer indoor residual spraying (Uganda MoH, 2015). ${ }^{29}$

The challenge of drug stock out at facilities and with CBDs was also confirmed by the Global fund auditors that found that 854 community based drug distributors (CBDs) from two sub-recipients to population services international (PSI) had anti-malarial medicine stock outs in the last quarter of $2014 .^{30}$ Though only $36 \%$ of the tests were positive for malaria, the percentage could be higher because the RDT used was specific for plasmodium falciparum. Other species of plasmodium like plasmodium malariae, ovale and vivax among others would still be positive from a blood slide. A fever that tests negative with RDT was then referred to the health facility for further investigations and treatment. More research is therefore needed to confirm the diagnosis of febrile illness that test negative with RDT. Secondly the same research needs to be repeated during the peak of the rainy season when the malaria cases are very high.

\section{Conclusion}

Stock of medicines remains a big challenge to the integrated community based management of malaria, primarily due to irrational use of up to $64 \%$ of the available ACTs. Unfortunately the stock outs are likely to be during the peak of rainy season when the accessibility to health facilities is a challenge and malaria is also at its peak. This causes the morbidity and mortality due to malaria to remain high despite the concerted efforts from the Ministry of health, UN agencies, international organizations and the local communities to fight malaria. The solution will be to train all Community based distributors on use of RDTs and provide them with regular supplies. Include other preventive measures like indoor residual spraying, health education and long lasting insecticide treated net use if the morbidity and mortality due to malaria can be significantly reduced in South Sudan.

\section{References}

[1].ALIMA 2017, South Sudan: Mobile clinics respond to malaria peak. South-Sudan-mobile-clinics-respondmalaria-peak, para 4

[2].Armah, M. Amaah, P. and Ebrahim M.J. 2016. Health cluster bulletin \# 406 September 2016. South Sudun. health-cluster-bulletin_24-august---06-sept-2016.pd

[3].DFID, 2015. Annual review summary sheet. Integrated Community Case Management (ICCM) South Sudan. South-Sudan-ICCM-Integrated-Community-Case-Management-DFID-2015-review.pdf?x30250

[4].Dick, M., Farai, C., \&Joseph, N. 2011. Pharmaceutical logistics assessment in South Sudan, Global health and technical assistance project. s19289en.pdf

[5].Global fund 2015; Audit report for the Global Fund Grants to South Sudan. Office of the inspector general, 5th October Geneva 2015 OIG_GF-OIG-15-016_Report_en.pdf

[6].Malaria Journal201413:45. Doi.1475-2875-13-45

[7].Margaret B Eyobo, Adwok C Awur, Gregory Wani, Ahmed I Julla, Constantino D Remijo, Bakhit Sebit, Robert Azairwe, Othwonh Thabo, Edward Bepo, Richard L Lako, Lul Riek and Emmanuel Chanda, 2014. Malaria indicator survey 2009, South Sudan: baseline results at household level

[8].Marycelina Mubi, Annika Janson, Marian Warsame, Andreas Mårtensson, Karin Källander, Max G. Petzold, Billy Ngasala, Gloria Maganga, Lars L. Gustafsson, Amos Massele, Göran Tomson, Zul Premji, and Anders Björkman, 2011. Malaria Rapid Testing by Community Health Workers Is Effective and Safe for Targeting Malaria Treatment: Randomised Cross-Over Trial in Tanzania doi.org/10.1371/journal.pone.0019753.

[9].Max Gibson and Eric Kramak, 2016. Understanding flood resilience in rural South Sudan. id=77f24aa163aa-4224-a69f-b5aa50d449b0.

[10]. MC, 2012. Integrated Community Case Management (ICCM) for Malaria, Pneumonia, Diarrhoea \& Severe Acute Malnutrition. Facilitator-Training-Manual-iCCM-South-Sudan_Malaria-Consortium_2012.pdf

\footnotetext{
${ }^{29}$ Uganda malaria indicator survey 2014-2015

${ }^{30}$ OIG_GF-OIG-15-016_Report_en.pdf

SIAPS, 2016. Annual Report: Program Year 5 October 2015-September 2016 2015-2016. pdf.usaid.gov/pdf_docs/PA00MW5Q.pdf
} 
DOI: $10.21522 /$ TIJPH.2013.06.02.Art002

ISSN: $2520-3134$

[11]. MoH, 2010. Ntegrated Community Case Management of Childhood Malaria, Pneumonia and Diarrhoea: Implementation Guidelines.

[12]. MoH, 2015. Uganda malaria indicator survey 2014-15. MIS21.pdf.

[13]. MOH, Republic of South Sudan, 2012. Health sector development plan 2012-2016. health-sectordevelopment-plan-2012-2016.pdf.

[14]. NBS, 2011. 25th November 2010 Key Indicators for Northern Bahr El Ghazal (N.B.G) key_indicators_for_northern_bahr_el_ghazal.pdf.

[15]. NMCP, 2015. South Sudan Malaria Case Management Guidelines and Training Manual ApprovedPrinted-Version_SSD-MCM-Guidelines_2015.pdf.

[16]. Ohnmar, Tun-Min, San-Shwe, Than-Win and Virasakdi Chongsuvivatwong 2012. Effects of malaria volunteer training on coverage and timeliness of diagnosis: a cluster randomized controlled trial in Myanmar.

[17]. PSI, 2014. Integrated Community Case Management of Pneumonia, Malaria \& Diarrhea. iCCM_Program_Brief.pdf.

[18]. SIAPS, 2015. Systems for Improved Access to Pharmaceuticals and Services: Malaria Quarterly Updatemalaria-pmi-quarterly-updates-april-june-2015.

[19]. SIAPS, 2016. Annual Report: Program Year 5 October 2015-September 2016. PA00MW5Q.pdf.

[20]. Uganda Malaria indicator survey 2009. Uganda Bureau of statistics, Kampala Uganda.

[21]. UNICEF, 2012. WHO/UNICEF joint statement Integrated Community Case Management (iCCM). statement_child_services_access_whounicef.pdf.

[22]. USAID 2011, Pharmaceutical logistics assessment in South Sudan, Global health and technical assistance project.

[23]. WHO Afro, 2017. South Sudan national malaria strategy 2014-2015. south-sudan-national-malariastrategic-plan-2014-2021-launched.

[24]. WHO Malaria Policy Advisory Committee and Secretariat Malar J (2016) 15:117.

[25]. WHO Malaria Policy Advisory Committee and Secretariat, 2016. Malaria Policy Advisory Committee to the WHO: conclusions and recommendations of eighth biannual meeting (September 2015). Malaria Journal201615:117 doi.s12936-016-1169-x.

[26]. WHO, 2010. How to use rapid diagnostic test (RDT).generic_PfPan_training_manual_web.pdf

[27]. WHO, 2010. Parasitological confirmation of malaria diagnosis: WHO technical consultation, Geneva, 6-8 October 2009. Doi.9789241599412_eng.pdf.

[28]. WHO, 2011. WHO Global Malaria Programme: World malaria report 2011. Doi. 9789241564403_eng.pdf.

[29]. WHO, 2012. Training module on malaria control: case management guide for participants. Doi.9789241503976_eng.pdf.

[30]. WHO, 2015. Global technical strategy for malaria 2016-2030. 9789241564991_eng.pdf.

[31]. World health organization, 2017. World malaria report 2017. 9789241565523-eng.pdf. 